\title{
Effects of ACE2 and Co-factors on the Mechanism of SARS-CoV-2 Infection Among Colorectal Cancer Patients
}

\section{Shuwei Li}

Nanjing Medical University

\section{Silu Chen}

Nanjing Medical University

Qiuyuan Zhu

Nanjing Medical University

Shuai Ben

Nanjing Medical University

Fang Gao

Nanjing Medical University

Junyi Xin

Nanjing Medical University

Mulong Du

Nanjing Medical University

Haiyan Chu

Nanjing Medical University

Dongying Gu

Nanjing Medical University

Zhengdong Zhang

Nanjing Medical University

Meilin Wang ( $\nabla$ mwang@njmu.edu.cn )

Nanjing Medical University

\section{Research}

Keywords: SARS-CoV-2, colorectal cancer, ACE2, susceptibility, prognosis

Posted Date: September 13th, 2021

DOI: https://doi.org/10.21203/rs.3.rs-868323/v1 
License: (c) (i) This work is licensed under a Creative Commons Attribution 4.0 International License. Read Full License 


\section{Abstract}

Background: The infection of host cells by SARS-CoV-2 is mediated by ACE2 and its co-factors, including TMPRSS2 and FURIN. Patients with cancer are highly susceptible to COVID-19 and exhibit a higher risk of severe progression. However, the mechanism of SARS-CoV-2 infection on colorectal cancer prognosis is largely unknown. Here, we investigated the role of key genes related to SARS-CoV-2 in colorectal cancer.

Methods: RNA sequencing (RNA-Seq), proteomics and single-cell RNA-Seq were used to profile the expression of ACE2, TMPRSS2, and FURIN in colorectal cancer. A pseudovirus was used to transfect colon cells to compare the infection rate of SARS-CoV-2 between different cell types. The TIMER database was used to analyze the association of candidate genes with immune infiltration in colorectal cancer. Cox regression model was performed to evaluate genetic effects of ACE2 on colorectal cancer prognosis.

Results: ACE2 was upregulated in colorectal cancer tissues, while TMPRSS2 and FURIN were downregulated. Increased ACE2 expression was significantly associated with a decreased tumor mutational burden. Furthermore, ACE2 promoted the SARS-CoV-2 infection in colon cancer cells than that in normal colon epithelial cells, as well as that $A C E 2$ could affected the immune infiltration level and prognosis in colorectal cancer patients. Moreover, individuals with genetic variants in ACE2 exhibited poor overall survival of colorectal cancer.

Conclusions: These results are the first time to identify that ACE2 and its co-factors are associated with the infection and survival of colorectal cancer patients with SARS-CoV-2. Our findings would be helpful for extending the mechanisms in SARS-CoV-2 infection among colorectal cancer.

\section{Background}

Severe acute respiratory syndrome coronavirus 2 (SARS-CoV-2), a novel coronavirus leading to coronavirus disease (COVID-19), is a new major disease threat to humans. The rapid spread of COVID-19 in approximately 200 countries and territories has created a global epidemic affecting more than 200 million patients in most countries of the world, with more than four million confirmed deaths.

Recent studies have reported that patients with cancer show a higher risk of infection with SARS-CoV-2, more severe adverse events and a poorer prognosis than those without cancer [1, 2]. Notably, cancer history is the only independent risk factor for COVID-19 among common comorbidities [3]. This is due to the systemic immunosuppressive state caused by several anticancer treatments $[4,5]$. Lymphopenia or neutropenia in cancer patients could also cause worse outcomes of COVID-19 [6, 7]. Colorectal cancer is one of the most common cancers and is the second most common cause of cancer-related mortality [8]. As reported recently, the levels of IL-6, numbers of neutrophils and ratio of neutrophils to lymphocytes (NLR) in the mild group are significantly lower than those in the severe group of colorectal cancer patients with COVID-19 [9]. In addition, SARS-CoV-2 can infect intestinal organoids according to the identification 
of live virus in stool samples from COVID-19 patients $[10,11]$. However, there is still a large gap in the understanding of biological mechanisms of colorectal cancer in association with SARS-COV-2.

SARS-CoV-2 was previously shown to enter host cells by binding to angiotensin-converting enzyme 2 (ACE2) $[12,13]$. ACE2 can cleave both angiotensin 1 and 2 , which is the only experimentally confirmed SARS-CoV-2 receptor $[14,15]$. Additionally, transmembrane protease serine 2 (TMPRSS2) and FURIN (FURIM), which are two co-factors of ACE2, can facilitate the cleavage of the SARS-CoV-2 spike glycoprotein $[12,16]$. Although ACE2 and its co-factors have been reported to be differentially expressed in various tissues to regulate the susceptibility of cancer patients to SARS-CoV-2 infection $[17,18]$, the expression and functional role of $A C E 2$ and its co-factors in colorectal cancer remain to be explored.

In this study, we explored the expression pattern of $A C E 2$ and its co-factors at mRNA and protein level in colorectal cancer, as well as their effects on infection rate of SARS-CoV-2 between colorectal cancer and normal colon epithelial cells. Furthermore, the regulation of immune inflammation and the genetic effects of ACE2 and its co-factors were analyzed to identify the mechanisms of host factors in the induction of SARS-CoV-2 infection and ultimately provide treatment strategies for SARS-CoV-2 infections in colorectal cancer patients.

\section{Methods}

\section{Study populations and samples}

All the participants in this study were unrelated Han Chinese individuals and provided written informed consent. The detailed information of this study population is described in the Supplementary Materials and Methods. In addition, a total of 544 patients were recruited for a follow-up investigation performed via telephone every six months. The characteristics of the subjects are shown in Table S1. This study was approved by the institutional review board of Nanjing Medical University, China.

\section{RNA isolation and RNA sequencing (RNA-Seq)}

Total RNA was extracted using TRIzol Reagent (Invitrogen, CA, USA) according to the manufacturer's protocol. The concentration and integrity of RNA were confirmed using a Nanodrop 2000 spectrometer (ThermoFisher) and an Agilent 2100 Bioanalyzer (Agilent, CA, USA). The detailed protocol for RNA-Seq is described in the Supplementary Materials and Methods.

\section{Protein extraction and tryptic digestion for tandem mass tag (TMT) analysis}

The collected tissue samples were fixed in RNAlater stabilization solution (Invitrogen, Carlsbad, CA) and stored in a $-80^{\circ} \mathrm{C}$ refrigerator for protein extraction. The detailed protocols for protein extraction and the 
TMT experiment are described in the Supplementary Materials and Methods.

\section{Gene expression analysis in different tissues}

mRNA expression profiles were downloaded from datasets of The Cancer Genome Atlas (TCGA) (http://cancergenome.nih.gov/) [19], Gene Expression Omnibus (GEO) and Gene Expression Profiling Interactive Analysis (GEPIA) [20]. Immunohistochemical images and protein expression profiles were obtained from the Human Protein Atlas (HPA) database [21]. The single-cell RNA-Seq data for colorectal cancer tissues were obtained from GSE81861 [22]. The details of the data sources and the processing methods for the gene expression analysis are provided in the Supplementary Materials and Methods.

\section{Cell transfection and SARS-CoV-2 pseudovirus treatment experiments}

The details of the cell lines and cell cultures are described in the Supplementary Materials and Methods. For the stable overexpression of ACE2, cells were transfected with the GV358 lentivirus vector or a negative control (GeneChem, China) expressing green fluorescent protein (GFP) as a marker. The stable effect of ACE2 overexpression was confirmed by the observation of GFP under a fluorescence microscope and Western blotting. The SARS-CoV-2 pseudovirus was produced with the pLVX-EGFPLuciferase reporter. To evaluate the infection ability and rate of SARS-CoV-2 in different types of colon cells, FHC, HCoEpiC, SW620 and LoVo cells were used to conduct luciferase activity assays and immunofluorescence staining after the stable overexpression of ACE2. The protocols for these experimental procedures are available in the Supplementary Materials and Methods.

\section{Tumor Immune Estimation Resource (TIMER) analysis}

TIMER is a web server for the systematic analysis of immune infiltrates in different cancers [23, 24]. This comprehensive resource provides six major analytic modules for exploring the associations between immune infiltrates and different factors, such as gene expression, somatic mutation and clinical outcomes. We analyzed the association of the expression of selected genes with the abundance of infiltrating immune cell types. Expression scatter plots between selected genes were generated using the correlation module, together with Spearman's correlation and the estimated statistical significance.

\section{Genetic variants and somatic mutation analysis}

The details of the SNP genotyping methods and the somatic mutation analyses are described in the Supplementary Materials and Methods. 


\section{Statistical analysis}

All continuous data are presented as the mean \pm SD. A two-sided $P$ value of less than 0.05 was regarded as statistically significant. The more details of statistical analysis are described in the Supplementary Materials and Methods.

\section{Results}

\section{Expression pattern of ACE2, TMPRSS2 and FURIN across different colorectal tissues}

We first assessed the mRNA expression pattern of ACE2 in colorectal cancer tumors using in-house RNASeq, and found that the mRNA expression level of ACE2 was significantly higher in tumor tissues than in adjacent normal tissues (Figure 1A). However, the distinct expression was not validated in the TCGA database (Figure S1A); and even could not be found in meta-analysis of ACE2 expression using the combined in-house RNA-Seq dataset, TCGA and GEO datasets (Figure S1B). Therefore, we used TMTbased global proteomics to evaluate the protein expression of ACE2 in paired colorectal clinical tissues. The protein expression of ACE2 was higher in colorectal tumor tissues than in paired normal tissues (Figure 1B), which was also supported by immunohistochemical images that high staining and strong intensity of ACE2 existed in colon and rectum tumor tissues (Figure 1C). Subsequently, we analyzed the mRNA expression of TMPRSS2 and FURIN between colorectal cancer tissues and normal tissues via a meta-analysis of the RNA-Seq results and TCGA and GEO datasets. As shown in Figure 1D-E, the expression of both TMPRSS2 and FURIN was significantly decreased in colorectal cancer tissues compared with normal tissues. The immunohistochemical images of TMPRSS2 and FURIN staining also revealed that TMPRSS2 and FURIN were downregulated in colon tumor tissues (Figure 1F$\mathrm{G})$. Additionally, the heat map illustrated that these two genes were co-lower expressed in colorectal cancer tissues compared to paired normal tissues (Figure 1H). Furthermore, the expression levels of TMPRSS2 and FURIN gradually decreased along with malignant progression in the course of normal, adenoma and tumor (Figure 1I-L).

Given that ACE2 cooperating with co-factors TMPRRS2 and FURIN, plays key roles in regulating the binding and entry affinity of SARS-CoV-2, we further conducted Spearman's correction analysis between ACE2 and its co-factors. As shown in Figure S1C-D, the expression of ACE2 was positively correlated with TMPRRS2 and negatively corrected with FURIN. A positive correlation was also detected between the expression levels of the two co-factors (Figure S1E). Therefore, we hypothesized that three genes were coexpressed and interacted in colorectal tumor tissues.

We then examined the role of onset age in the expression of these three genes. Higher ACE2 expression and lower FURIN expression were significantly related to late-onset colorectal cancer, while no association was found between onset age and TMPRSS2 expression (Figure S2). We also found 
no significant differences in the tumor site or tumor stage related to the expression of these three genes (Figure S2).

\section{RNA and protein expression of ACE2 and its co-factors in pan-cancer tissues}

Based on the GEPIA2 and HPA databases, we compared the mRNA and protein expression of ACE2 and its co-factors in different tissues. Notably, FURIN mRNA showed high abundance in most types of tissues compared with that of ACE2 and TMPRSS2 (Figure S3). However, the protein immunoreactivity of TMPRSS2 in most types of tumor tissues was generally weaker or negative compared with that of ACE2 and FURIN (Figure S4). ACE2 protein expression was observed to show moderate to strong cytoplasmic and membranous immunoreactivity in colorectal and renal cancers.

\section{Co-expression pattern of $A C E 2$ and its co-factors in colorectal cancer cells determined by single-cell RNA-Seq analysis}

We next carried out single-cell RNA-Seq to evaluate the expression pattern of ACE2 and its co-factors in colorectal cancer tissues. As shown in Figure 2A-D, among colorectal tumor cell clusters, the expression of ACE2 and TMPRSS2 was heterogeneous, with some cells co-expressing both ACE2 and TMPRSS2 (9.33\%), while some tumor cell clusters expressed only ACE2 (3.2\%) or only TMPRSS2 (31.2\%). Similarly, $15.2 \%$ of colorectal tumor cells co-expressed both ACE2 and FURIN, while $9.87 \%$ and $2.67 \%$ of cell clusters expressed only ACE2 or only TMPRSS, respectively (Figure 2E-H). These results provided the coexpression pattern of $A C E 2$ and the co-factors in the colorectal tumor cells.

\section{The infection rate of SARS-CoV-2 in colon cells}

To evaluate the infection rate of SARS-CoV-2 in colon cell lines, we first tested the ACE2 expression level of normal colon epithelial cells (FHC and HCoEpiC) and colon tumor cells (SW620 and LoVo). None of these cells showed ACE2 expression that was detectable by Western blotting. Therefore, cell lines with stable overexpression of $A C E 2$ were cultured, and the transduction efficiency was confirmed by GFP fluorescence and Western blotting (Figure 3A-B). Subsequently, we transfected the SARS-CoV-2 pseudovirus into cells and performed luciferase reporter assays. We found that relative luciferase activity was significantly increased in colorectal tumor cells compared with normal colon epithelial cells (Figure 3C). Finally, higher positive staining of SARS-CoV-2 was detected in colorectal tumor cells by immunofluorescent staining, while lower positive staining was observed in normal colon epithelial cells (Figure 3D). For the first time, we identified a higher infection rate of SARS-CoV-2 in ACE2-positive colorectal tumor cells than in normal colon epithelial cells. 


\section{Association of $A C E 2$ and co-factor expression with immune infiltration in colorectal cancer tissues}

Given the association of crucial gene expression with immune infiltration levels in colorectal cancer, we investigated whether ACE2 and its co-factors were associated with immune infiltration in different types of immune cells by using the TIMER algorithm (Table S2). We observed a significant negative association of tumor purity with FURIN $\left(r=-0.180, P=2.58 \times 10^{-4}\right)$, but no significant association of tumor purity with ACE2 or TMPRSS2 was found (Figure 4). Interestingly, we found that ACE2 expression

presented a significant negative association with the infiltrating levels of neutrophils $(r=-0.115, P=2.11$ $\left.\times 10^{-2}\right)$ and dendritic cells $\left(r=-0.097, P=5.16 \times 10^{-2}\right)$ in colon cancer tissues (Figure 4). Moreover, the expression of TMPRRS2 and FURIN presented a significant positive association with the infiltrating levels of neutrophils $\left(r=0.124, P=1.29 \times 10^{-2}\right.$ in TMPRSS2; $r=0.225, P=5.12 \times 10^{-6}$ in FURIM $)$ and dendritic cells $\left(r=0.121, P=1.53 \times 10^{-2}\right.$ in TMPRSS2; $r=0.288, P=4.20 \times 10^{-9}$ in FURIM $)$ in colon cancer tissues (Figure 4). These findings revealed that ACE2 and its co-factors play specific roles in immune infiltration in colorectal cancers, especially in of neutrophil and dendritic cells.

\section{Prognostic analysis of $A C E 2$ and its co-factors in colorectal cancer tissues}

To explore the prognostic value of ACE2 and its co-factors in colorectal cancer, we performed KaplanMeier analysis based on the TCGA database. We found that low expression of ACE2 was significantly associated with a poor overall survival time $(P=0.028)$ (Figure $5 A)$, while no significant association was observed between the overall survival time and the expression levels of TMPRSS2 $(P=0.073)$ or FURIN $(P$ $=0.220$ ) (Figure 5B-C). In addition, the overall survival time of patients in the higher-risk-score group was obviously longer than that of patients in the low risk score group for the combination of ACE2 and FURIN $(P=0.046)$ (Figure 5D). However, we only detected a weak association between a longer overall survival time and the higher risk score group for the combination of ACE2 and TMPRSS2 $(P=$ 0.069) (Figure $5 \mathrm{E}$ ) or the combination of three genes $(P=0.058)$ (Figure $5 \mathrm{~F}$ ). We also visualized the association between survival status and risk score rank as well as overall survival (Figure 5G-I).

\section{Association of genetic variants in ACE2 and its co-factors with colorectal cancer risk and survival}

Given that genetic variants in cancer-related genes are associated with colorectal cancer, we sought to investigate the genetic effect of SNPs in ACE2 and its co-factors on colorectal cancer susceptibility and prognosis. A summary of the experimental design and workflow is shown in Figure S5. A total of 19 candidate SNPs in ACE2 and its co-factors were included in the genotyping analysis after quality control 
and function prediction. Among these SNPs, no significant association was detected between candidate SNPs and the susceptibility of colorectal cancer (Table S3).

We then used 540 patients with follow-up information to confirm the genetic effect of candidate SNPs in predicting the prognosis of colorectal cancer (Table S4). Only two SNPs in the ACE2 gene were significantly associated with colorectal cancer survival time (HR $=1.40, P=0.017$ for rs2106809 and HR $=1.34, P=0.038$ for rs2285666) (Figure 6A-B). We also combined these two SNPs according to the number of risk alleles. As shown in Figure $6 \mathrm{C}$, colorectal cancer patients with 1-4 risk alleles presented significantly poorer overall survival than those with 0 risk alleles $(P<0.05)$. Due to these two SNPs located in the ACE2 gene, we performed the functional annotation of rs2106809 and rs2285666 and the SNPs showing high linkage disequilibrium (rs4646142 and rs184697926) based on the Encyclopedia of DNA Elements (ENCODE) and Roadmap Epigenome Projects. The region encompassing these four SNPs was predicted in silico to present possible enhancer activity (Figure 6D), which indicated that genetic variants in the $A C E 2$ gene could be useful in prognosis prediction for colorectal cancer.

\section{Somatic mutation patterns of $A C E 2$ and its co-factors in colorectal cancer tissues}

We extracted mutational signatures from the TCGA database to identify somatic mutation patterns in ACE2 and its co-factors in colorectal cancer patients. As demonstrated in Figure S6A and Table S5, missense mutations in ACE2 (73.33\%), FURIN (75.00\%) and TMPRSS2 (83.33\%) are particularly common in colorectal cancer tissues. Interestingly, we observed a significant increase in the mutation frequency of the ACE2 gene in the early-onset colorectal cancer group compared with the late-onset colorectal cancer group ( $P=0.023$ ) (Table S6). We also detected a decreasing trend in ACE2 expression in patients with ACE2 mutations $(P=0.059)$ (Figure S6B).

TMB is an emerging biomarker for the immunotherapy response of cancers. In this study, a higher TMB was detected in individuals with ACE2 mutations compared with those without $A C E 2$ mutations $(P=3.32$ $\left.\times 10^{-5}\right)$ (Figure S6C). We also found a significant decrease in TMB associated with increased ACE2 expression $(r=-0.149, P<0.001)$ (Figure S6D). Collectively, these results highlight the important roles of somatic mutations in the ACE2 gene and TMB in colorectal cancer progression.

\section{Discussion}

The ongoing outbreak of COVID-19 has affected more than 200 countries. Patients with cancer are highly susceptible to COVID-19 and show a higher risk of severe adverse events and death $[2,25]$. However, there is limited available information about the mechanisms underlying the outcomes of colorectal cancer patients who contract COVID-19. In this study, we found that ACE2 was upregulated, while TMPRSS2 and FURIN were downregulated in colorectal cancer tissues. In addition, the infection rate of SARS-CoV-2 was 
significantly higher in ACE2-positive colon tumor cells. The expression of three genes was associated with the immune infiltration level and the overall survival time of colorectal cancer patients. Genetic factors related to $A C E 2$ were also found to play key roles in colorectal cancer prognosis. Our study findings suggest that $A C E 2$ and its co-factors participate in the mechanisms underlying the association of colorectal cancer with COVID-19 (Fig. 7).

SARS-CoV-2, a single-stranded positive-sense RNA virus of the $\beta$-Coronaviridae family, has been confirmed to enter different mammalian cell types showing the expression of the ACE2 receptor [26, 27]. $A C E 2$ is abundantly expressed in digestive tract organs $[28,29]$. Recent research suggested that the expression of $A C E 2$ gradually increases from the normal colon epithelium to adenoma and colorectal tumor tissues [30]. We found that ACE2 was upregulated in colorectal cancer tissues and was related to the overall survival time of colorectal cancer patients. SARS-CoV-2 can interact with ACE2 to infect host cells via the $S 1$ receptor-binding domain (RBD) of its surface spike glycoprotein [12,13]. Previous studies have used authentic and pseudovirus SARS-CoV-2 neutralization assays to obtain antibodies that can significantly protect host cells from SARS-CoV-2 infection [31-33]. Moreover, SARS-CoV-2 could bind platelet ACE2 to induce thrombosis in COVID-19 [34]. We found that colon cancer cells are more likely to be infected by SARS-CoV-2 than normal colon epithelial cells. This provides novel evidence for understanding the susceptibility and prognosis of colorectal cancer patients after infection with COVID19.

TMPRSS2 and FURIN are the major proteases that cleave the SARS-CoV-2 spike glycoprotein [12, 35]. A previous study showed that the entry of SARS-CoV-2 could be successfully blocked by blocking the protease activity of TMPRSS2 in colon cells [12], which indicated that SARS-CoV-2 requires other cofactors, including TMPRSS2 and FURIN. Moreover, the cleavage of the spike glycoprotein at the FURIN cut site could induce the binding ability of SARS-CoV-2 to ACE2 [36]. Notably, TMPRSS2 and FURIN have special impacts on the cellular neighborhood, significantly increasing the susceptibility of ACE2-positive cell clusters to infection with SARS-CoV-2 [37]. According to our data, TMPRSS2 and FURIN are downregulated in colorectal cancer tissues. In addition, the co-expression of these two genes with ACE2 may show potential for predicting the overall survival of colorectal cancer patients. However, the involvement of ACE2 and its co-factors in the infection pathway of SARS-CoV-2 remains to be investigated by future experimental methods.

Recent studies have suggested that severe adverse events in COVID-19 patients are dramatically related to an increase in innate immune cells such as neutrophils, indicating that immunopathology is involved $[38,39]$. Previous in vivo experiments confirmed that ACE2 suppresses lung inflammation and neutrophil infiltration by reducing the activity IL-17 signaling [40]. In addition, significant positive and negative correlations have been confirmed between ACE2 expression and IL-17(Th17)-dependent and Th2dependent epithelial gene signatures, respectively [41, 42]. Interestingly, our data demonstrated that ACE2 was significantly negatively correlated with the infiltrating levels of neutrophils and dendritic cells. These findings may explain the association of abnormal immune infiltration levels with the outcomes of COVID19-infected colorectal cancer patients. 
Genetic variants in cancer-related genes are associated with the risk and prognosis of cancer. Numerous studies on genetic variants in ACE2 have focused on its association with cardiovascular disease and stroke $[43,44]$, but there is a lack of reports on its relationship to colorectal cancer. Our study indicated that rs2106809 and rs2285666 in ACE2 were associated with the overall survival of colorectal cancer patients. Bioinformatics analysis demonstrated that rs 2106809 could create an intronic exonic splicing enhancer (ESE) site to alter the splicing efficiency of ACE2 [45]. In addition, the SNP rs2285666, which is located in a splice region of $A C E 2$, might show strong functional regulation of $A C E 2$. TMB, in concert with PD-L1 expression, is emerging as a potential biomarker. Patients with a high TMB have been observed to present an association with the response to PD-1/PD-L1 blockade, including melanoma and colorectal cancer $[46,47]$. Chan et al. identified TMB development as an immunotherapy biomarker across several cancer types [48]. In addition, TMB is associated with the prognosis and the response to immune checkpoint inhibitors in colorectal cancer patients $[49,50]$. Our results further identified a significant decrease in TMB associated with increased ACE2 expression, which suggested that a higher TMB shows a significant correlation with colorectal cancer progression.

\section{Conclusions}

Taken together, this is the first study to identify that higher expression of ACE2 and lower expression of its co-factors significantly increase the susceptibility of ACE2-positive colorectal cancer cells to SARS-CoV-2 infection. These genes are also associated with abnormal immune infiltration levels and the prognosis of colorectal cancer patients. Our findings could be helpful for further exploring the mechanism underlying the association of colorectal cancer with COVID-19.

\section{Abbreviations}

SARS-CoV-2: severe acute respiratory syndrome coronavirus 2; COVID-19: coronavirus leading to coronavirus disease; WHO: World Health Organization; NLR: neutrophils to lymphocytes; ACE2: angiotensin-converting enzyme 2; TMPRSS2: transmembrane protease serine 2; RNA-Seq: RNA sequencing; TMT: tandem mass tag; TCGA: The Cancer Genome Atlas; GEO: Gene Expression Omnibus; GEPIA: Gene Expression Profiling Interactive Analysis; HPA: Human Protein Atlas; GFP: green fluorescent protein; TIMER: Tumor Immune Estimation Resource; SNPs: single nucleotide polymorphisms; WES: whole-exome sequencing; TMB: tumor mutation burden.

\section{Declarations}

\section{- Ethics approval and consent to participate}

This study was approved by the institutional review board of Nanjing Medical University. 


\section{- Consent for publication}

All authors consented for publication.

\section{- Availability of data and materials}

Not applicable

\section{- Competing interests}

The authors declare that they have no competing interests.

\section{- Funding}

Not applicable.

\section{- Authors' contributions}

M.W. and Z.Z. designed the study and reviewed the manuscript; S.L., S.C. and Q.Z. performed statistical analysis, conducted experiments and wrote the manuscript; S.B., F.G. and J.X. contributed to technical and material support. M.D., H.C. and D.G. reviewed data and provided critical commnents and suggestions.

\section{- Acknowledgements}

The authors would like to thank all study participants, researchers and clinicians who contributed to this study.

\section{References}

1. Liang W, Guan W, Chen R, Wang W, Li J, Xu K, et al. Cancer patients in SARS-CoV-2 infection: a nationwide analysis in China. Lancet Oncol. 2020;21(3):335-7.

2. Zhang L, Zhu F, Xie L, Wang C, Wang J, Chen R, et al. Clinical characteristics of COVID-19-infected cancer patients: a retrospective case study in three hospitals within Wuhan, China. Ann Oncol. 2020;31(7):894-901.

3. Meng Y, Lu W, Guo E, Liu J, Yang B, Wu P, et al. Cancer history is an independent risk factor for mortality in hospitalized COVID-19 patients: a propensity score-matched analysis. J Hematol Oncol. 2020;13(1):75.

4. Yang K, Sheng Y, Huang C, Jin Y, Xiong N, Jiang K, et al. Clinical characteristics, outcomes, and risk factors for mortality in patients with cancer and COVID-19 in Hubei, China: a multicentre, 
retrospective, cohort study. Lancet Oncol. 2020;21(7):904-13.

5. Zhang H, Wang L, Chen Y, Wu Q, Chen G, Shen X, et al. Outcomes of novel coronavirus disease 2019 (COVID-19) infection in 107 patients with cancer from Wuhan, China. Cancer. 2020;126(17):402331.

6. Goyal P, Choi JJ, Pinheiro LC, Schenck EJ, Chen R, Jabri A, et al. Clinical Characteristics of Covid-19 in New York City. N Engl J Med. 2020;382(24):2372-4.

7. Jee J, Foote MB, Lumish M, Stonestrom AJ, Wills B, Narendra V, et al. Chemotherapy and COVID-19 Outcomes in Patients With Cancer. J Clin Oncol. 2020:JC02001307.

8. Bray F, Ferlay J, Soerjomataram I, Siegel RL, Torre LA, Jemal A. Global cancer statistics 2018: GLOBOCAN estimates of incidence and mortality worldwide for 36 cancers in 185 countries. CA Cancer J Clin. 2018;68(6):394-424.

9. Ma J, Yin J, Qian Y, Wu Y. Clinical characteristics and prognosis in cancer patients with COVID-19: A single center's retrospective study. J Infect. 2020;81(2):318-56.

10. Wu Y, Guo C, Tang L, Hong Z, Zhou J, Dong X, et al. Prolonged presence of SARS-CoV-2 viral RNA in faecal samples. Lancet Gastroenterol Hepatol. 2020;5(5):434-5.

11. Wang W, Xu Y, Gao R, Lu R, Han K, Wu G, et al. Detection of SARS-CoV-2 in Different Types of Clinical Specimens. JAMA. 2020;323(18):1843-4.

12. Hoffmann M, Kleine-Weber H, Schroeder S, Krüger N, Herrler T, Erichsen S, et al. SARS-CoV-2 Cell Entry Depends on ACE2 and TMPRSS2 and Is Blocked by a Clinically Proven Protease Inhibitor. Cell. 2020;181(2):271-80. e8.

13. Walls AC, Park YJ, Tortorici MA, Wall A, McGuire AT, Veesler D. Structure, Function, and Antigenicity of the SARS-CoV-2 Spike Glycoprotein. Cell. 2020;181(2):281-92. e6.

14. Yan R, Zhang Y, Li Y, Xia L, Guo Y, Zhou Q. Structural basis for the recognition of SARS-CoV-2 by fulllength human ACE2. Science. 2020;367(6485):1444-8.

15. Zhang H, Penninger JM, Li Y, Zhong N, Slutsky AS. Angiotensin-converting enzyme 2 (ACE2) as a SARS-CoV-2 receptor: molecular mechanisms and potential therapeutic target. Intensive Care Med. 2020;46(4):586-90.

16. Coutard B, Valle C, de Lamballerie X, Canard B, Seidah NG, Decroly E. The spike glycoprotein of the new coronavirus 2019-nCoV contains a furin-like cleavage site absent in CoV of the same clade. Antiviral Res. 2020;176:104742.

17. Du M, Cai G, Chen F, Christiani DC, Zhang Z, Wang M. Multiomics Evaluation of Gastrointestinal and Other Clinical Characteristics of COVID-19. Gastroenterology. 2020;158(8):2298-301. e7.

18. Kong Q, Xiang Z, Wu Y, Gu Y, Guo J, Geng F. Analysis of the susceptibility of lung cancer patients to SARS-CoV-2 infection. Mol Cancer. 2020;19(1):80.

19. Weinstein JN, Collisson EA, Mills GB, Shaw KR, Ozenberger BA, Ellrott K, et al. The Cancer Genome Atlas Pan-Cancer analysis project. Nat Genet. 2013;45(10):1113-20. 
20. Tang Z, Li C, Kang B, Gao G, Zhang Z. GEPIA: a web server for cancer and normal gene expression profiling and interactive analyses. Nucleic Acids Res. 2017;45(W1):W98-102.

21. Uhlén $M$, Fagerberg $L$, Hallström BM, Lindskog $C$, Oksvold P, Mardinoglu A, et al. Proteomics. Tissuebased map of the human proteome. Science. 2015;347(6220):1260419.

22. Li H, Courtois ET, Sengupta D, Tan Y, Chen KH, Goh JJL, et al. Reference component analysis of single-cell transcriptomes elucidates cellular heterogeneity in human colorectal tumors. Nat Genet. 2017;49(5):708-18.

23. Li B, Severson E, Pignon JC, Zhao H, Li T, Novak J, et al. Comprehensive analyses of tumor immunity: implications for cancer immunotherapy. Genome Biol. 2016;17(1):174.

24. Li T, Fan J, Wang B, Traugh N, Chen Q, Liu JS, et al. TIMER: A Web Server for Comprehensive Analysis of Tumor-Infiltrating Immune Cells. Cancer Res. 2017;77(21):e108-e10.

25. Dai M, Liu D, Liu M, Zhou F, Li G, Chen Z, et al. Patients with Cancer Appear More Vulnerable to SARSCoV-2: A Multicenter Study during the COVID-19 Outbreak. Cancer Discov. 2020;10(6):783-91.

26. Zhou P, Yang XL, Wang XG, Hu B, Zhang L, Zhang W, et al. A pneumonia outbreak associated with a new coronavirus of probable bat origin. Nature. 2020;579(7798):270-3.

27. Cui J, Li F, Shi ZL. Origin and evolution of pathogenic coronaviruses. Nat Rev Microbiol. 2019;17(3):181-92.

28. Zhang H, Kang Z, Gong H, Xu D, Wang J, Li Z, et al. The digestive system is a potential route of 2019nCov infection: a bioinformatics analysis based on single-cell transcriptomes. bioRxiv. 2020:2020.01.30.927806.

29. Wang J, Zhao S, Liu M, Zhao Z, Xu Y, Wang P, et al. ACE2 expression by colonic epithelial cells is associated with viral infection, immunity and energy metabolism. medRxiv. 2020:2020.02.05.20020545.

30. Chen H, Xuan B, Yan Y, Zhu X, Shen C, Zhao G, et al. Profiling ACE2 expression in colon tissue of healthy adults and colorectal cancer patients by single-cell transcriptome analysis. medRxiv. 2020.

31. Ju B, Zhang Q, Ge X, Wang R, Yu J, Shan S, et al. Potent human neutralizing antibodies elicited by SARS-CoV-2 infection. bioRxiv. 2020:2020.03.21.990770..

32. Liu X, Gao F, Gou L, Chen Y, Gu Y, Ao L, et al. Neutralizing Antibodies Isolated by a site-directed Screening have Potent Protection on SARS-CoV-2 Infection. bioRxiv. 2020:2020.05.03.074914.

33. Wang C, Li W, Drabek D, Okba NMA, van Haperen R, Osterhaus ADME, et al. A human monoclonal antibody blocking SARS-CoV-2 infection. bioRxiv. 2020:2020.03.11.987958.

34. Zhang S, Liu Y, Wang X, Yang L, Li H, Wang Y, et al. SARS-CoV-2 binds platelet ACE2 to enhance thrombosis in COVID-19. J Hematol Oncol. 2020;13(1):120.

35. Wu C, Liu Y, Yang Y, Zhang P, Zhong W, Wang Y, et al. Analysis of therapeutic targets for SARS-CoV-2 and discovery of potential drugs by computational methods. Acta Pharm Sin B. 2020;10(5):766-88.

36. Wrapp D, Wang N, Corbett KS, Goldsmith JA, Hsieh CL, Abiona O, et al. Cryo-EM structure of the 2019nCoV spike in the prefusion conformation. Science. 2020;367(6483):1260-3. 
37. Lukassen S, Chua RL, Trefzer T, Kahn NC, Schneider MA, Muley T, et al. SARS-CoV-2 receptor ACE2 and TMPRSS2 are primarily expressed in bronchial transient secretory cells. EMBO J. 2020;39(10):e105114.

38. Li X, Xu S, Yu M, Wang K, Tao Y, Zhou Y, et al. Risk factors for severity and mortality in adult COVID19 inpatients in Wuhan. J Allergy Clin Immunol. 2020;146(1):110-8.

39. Zhou F, Yu T, Du R, Fan G, Liu Y, Liu Z, et al. Clinical course and risk factors for mortality of adult inpatients with COVID-19 in Wuhan, China: a retrospective cohort study. Lancet. 2020;395(10229):1054-62.

40. Sodhi CP, Nguyen J, Yamaguchi Y, Werts AD, Lu P, Ladd MR, et al. A Dynamic Variation of Pulmonary ACE2 Is Required to Modulate Neutrophilic Inflammation in Response to Pseudomonas aeruginosa Lung Infection in Mice. J Immunol. 2019;203(11):3000-12.

41. Choy DF, Hart KM, Borthwick LA, Shikotra A, Nagarkar DR, Siddiqui S, et al. TH2 and TH17 inflammatory pathways are reciprocally regulated in asthma. Sci Transl Med. 2015;7(301):301ra129.

42. Bradding P, Richardson M, Hinks TSC, Howarth PH, Choy DF, Arron JR, et al. ACE2, TMPRSS2, and furin gene expression in the airways of people with asthma-implications for COVID-19. J Allergy Clin Immunol. 2020;146(1):208-11.

43. Yang M, Zhao J, Xing L, Shi L. The association between angiotensin-converting enzyme 2 polymorphisms and essential hypertension risk: A meta-analysis involving 14,122 patients. J Renin Angiotensin Aldosterone Syst. 2015;16(4):1240-4.

44. Wu X, Zhu B, Zou S, Shi J. The Association Between ACE2 Gene Polymorphism and the Stroke Recurrence in Chinese Population. J Stroke Cerebrovasc Dis. 2018;27(10):2770-80.

45. Chen YY, Liu D, Zhang P, Zhong JC, Zhang CJ, Wu SL, et al. Impact of ACE2 gene polymorphism on antihypertensive efficacy of ACE inhibitors. J Hum Hypertens. 2016;30(12):766-71.

46. Johnson DB, Frampton GM, Rioth MJ, Yusko E, Xu Y, Guo X, et al. Targeted Next Generation Sequencing Identifies Markers of Response to PD-1 Blockade. Cancer Immunol Res. 2016;4(11):95967.

47. Le DT, Uram JN, Wang $H$, Bartlett BR, Kemberling $H$, Eyring AD, et al. PD-1 Blockade in Tumors with Mismatch-Repair Deficiency. N Engl J Med. 2015;372(26):2509-20.

48. Chan TA, Yarchoan M, Jaffee E, Swanton C, Quezada SA, Stenzinger A, et al. Development of tumor mutation burden as an immunotherapy biomarker: utility for the oncology clinic. Ann Oncol. 2019;30(1):44-56.

49. Lee DW, Han SW, Bae JM, Jang H, Han H, Kim H, et al. Tumor Mutation Burden and Prognosis in Patients with Colorectal Cancer Treated with Adjuvant Fluoropyrimidine and Oxaliplatin. Clin Cancer Res. 2019;25(20):6141-7.

50. Schrock AB, Ouyang C, Sandhu J, Sokol E, Jin D, Ross JS, et al. Tumor mutational burden is predictive of response to immune checkpoint inhibitors in MSI-high metastatic colorectal cancer. Ann Oncol. 2019;30(7):1096-103. 


\section{Figure 1}

A
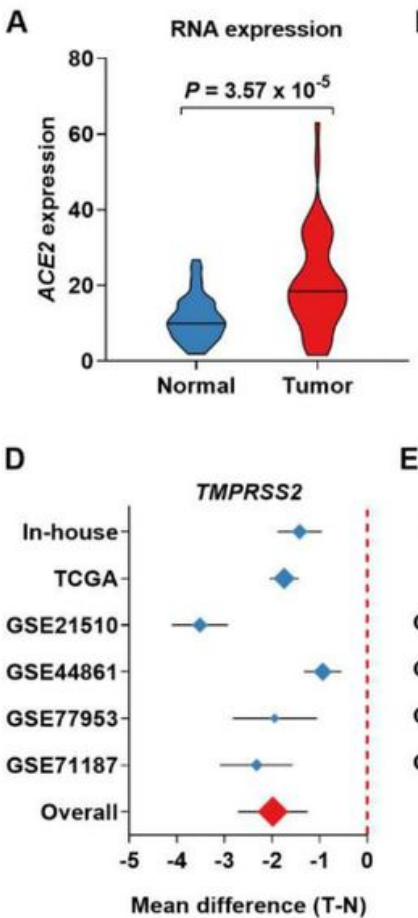

I

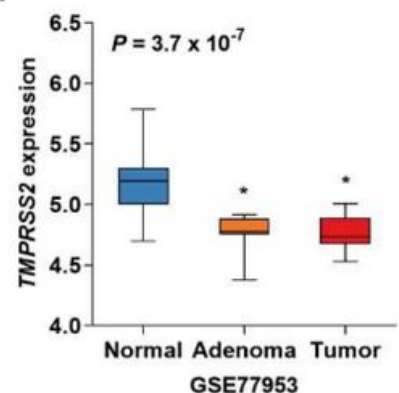

B

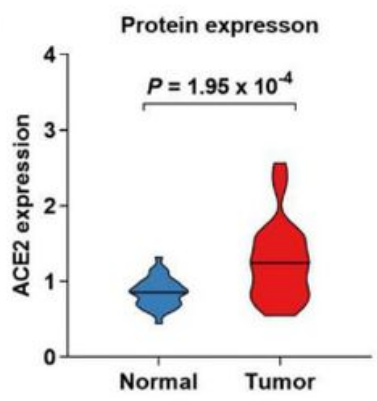

E

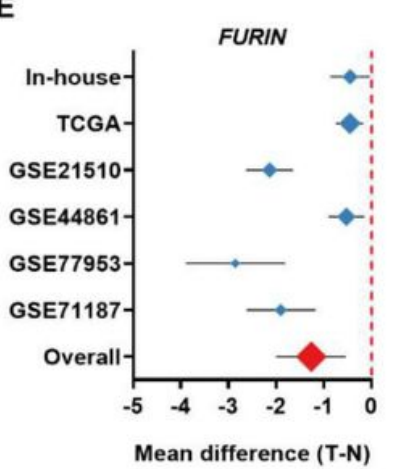

J

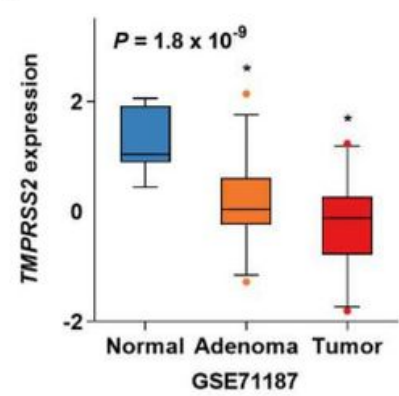

C

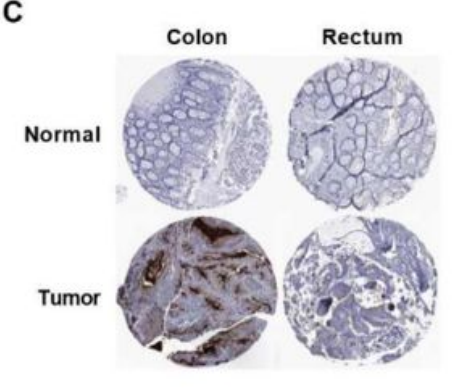

F

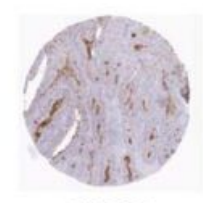

TMPRSS2

Tumorcells

Staining: Low Intensity: Moderat Quantity: $<25 \%$

G

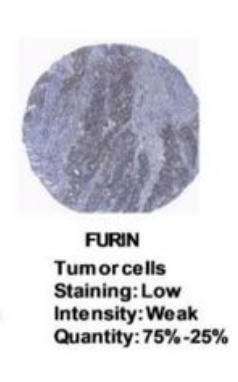

H

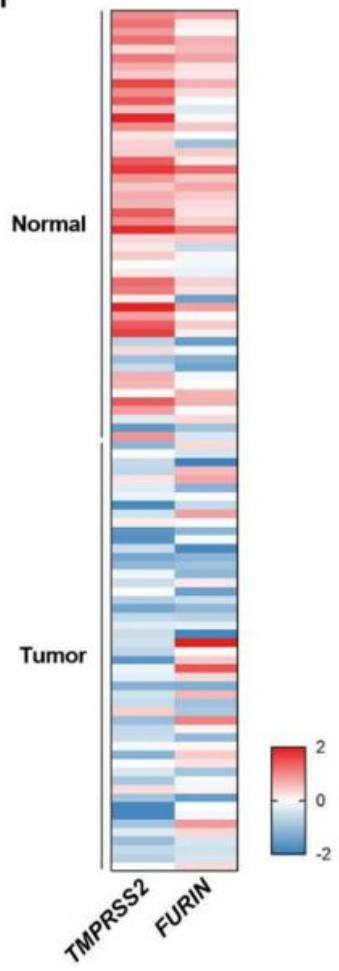

L

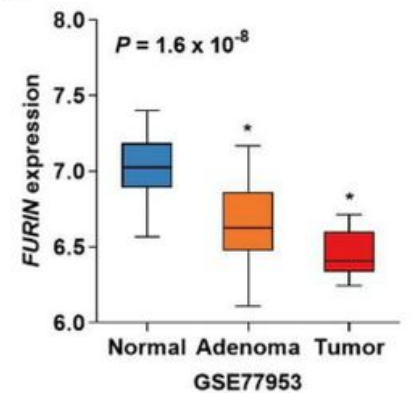

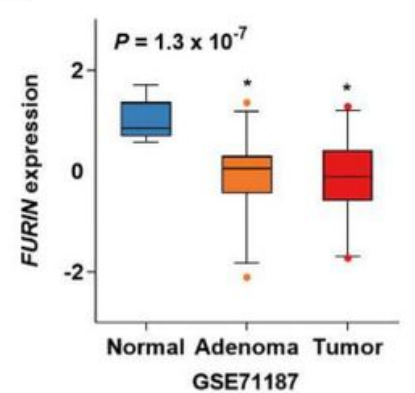

Figure 1

Differences in the expression of candidate genes between colorectal cancer tissues and normal tissues. (A) The mRNA expression of ACE2 determined from RNA-Seq. (B) The protein expression of ACE2 determined by proteomics. (C) Representative immunohistochemical images of ACE2 expression in colorectal tumor tissues from the HPA database. (D-E) Forest plots of TMPRSS2 mRNA expression (D) and FURIN mRNA expression (E). (F-G) Representative immunohistochemical images of TMPRSS2 (F) and FURIN (G) in colorectal tumor tissues from the HPA database. $(\mathrm{H})$ The heatmap of TMPRSS2 and 
FURIN mRNA expression from the TCGA database. (I-L) The box plot of TMPRSS2 and FURIN expression includes data from colorectal adenoma tissues from the GEO database.

\section{Figure 2}

A

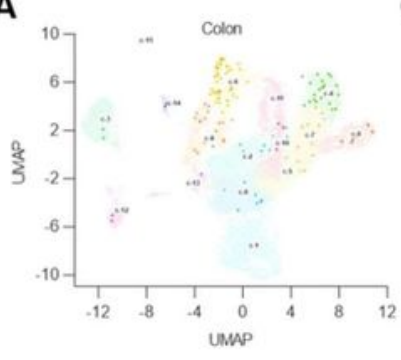

B

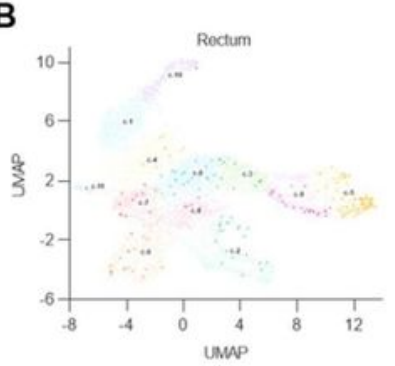

E

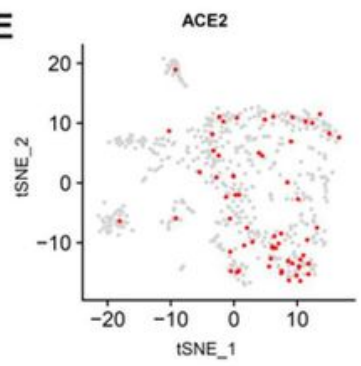

$\mathbf{F}$

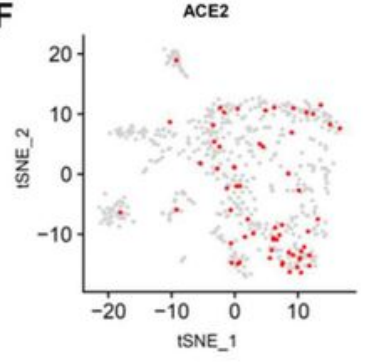

C

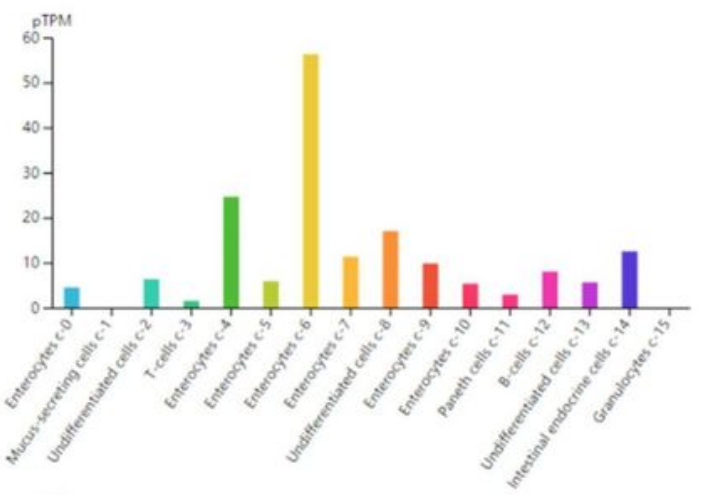

D

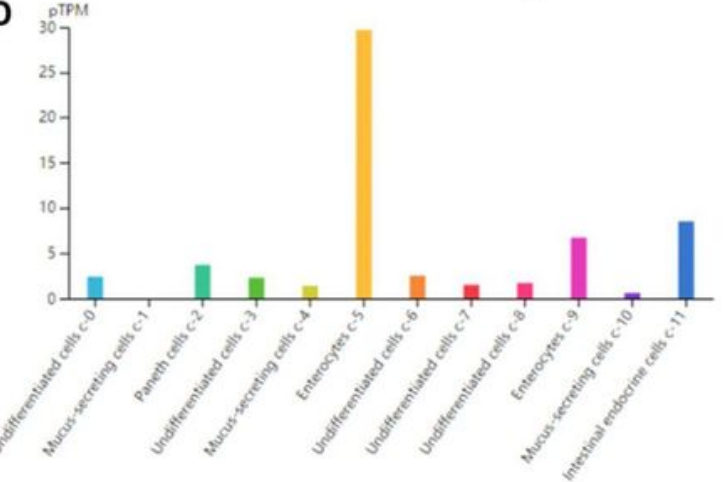

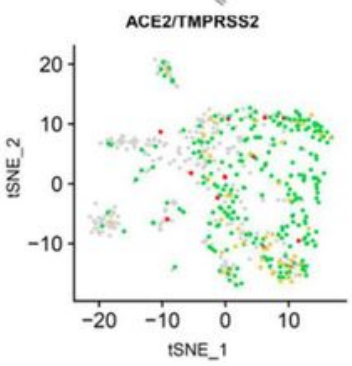

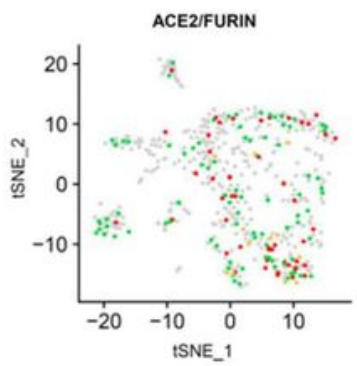

Ac-0 Enterocytes $(n=1729)$ c-1 Mucus-secreting cells $(n=1311$ ) c- -3 T-cells $(n=905)$ c. 4 Enterocytes ( $n=864$ c-5 Enterocytes $(n=813$ C.- Enterocytes (n=809 C.7 Enterocytes $(n=793)$
$c-8$ Undifferentiated cellis $(n=746)$
$c-9$ Enterocytes $(n=549)$ c-10 Enterocytes ( $n=495$ ) c-11 Paneth cells ( $n=354$ c-13 Undifterentated cells ( $n=149$ ) - 15 Granulocytes $(n=34)$
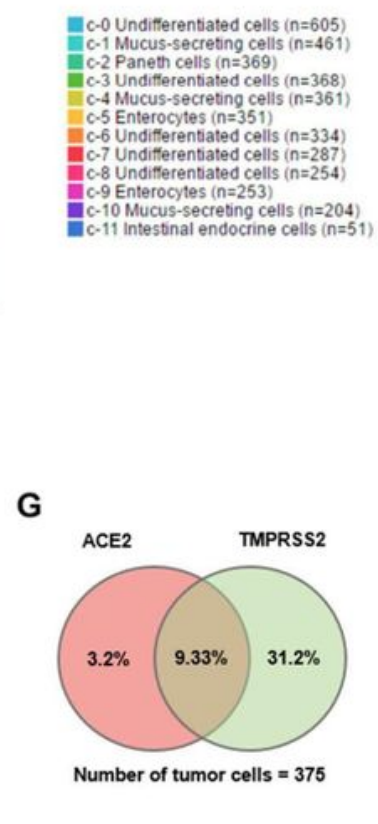

$\mathrm{H}$
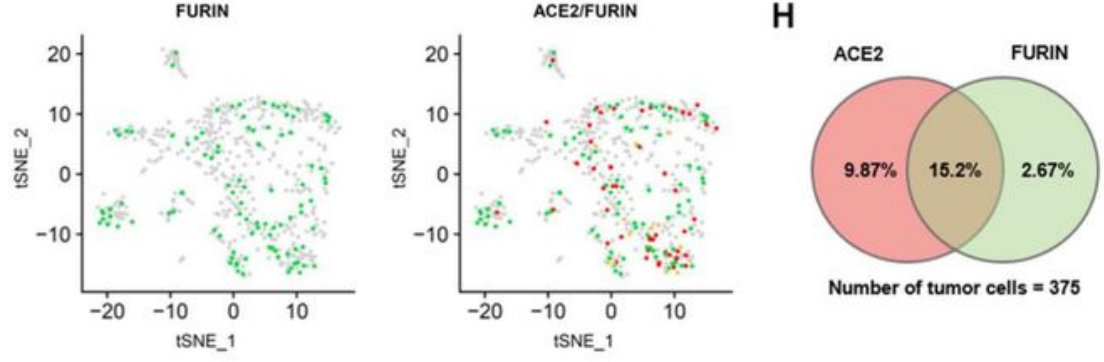

Figure 2

Expression analysis of ACE2 and TMPRSS2/FURIN by single-cell RNA-Seq in colorectal cancer cell clusters. (A-B) The UMAP plot of ACE2 RNA expression profile in colon (A) and rectum (B) tissues by single-cell RNA-Seq. (C-D) The Bar chart of ACE2 expression (pTPM) in each cell type cluster in colon (C) 
and rectum (D) tissues. (E-F) The distribution of ACE2, TMPRSS2 and FURIN in tumor cell clusters. (G) The combined distribution of ACE2 and TMPRSS2 in tumor cell clusters. ACE2 + , red. TMPRSS2 + , green. Both+, orange. Other, grey. $(\mathrm{H})$ The combined distribution of ACE2 (red) and FURIN (green) in tumor cell clusters. ACE2+, red. FURIN+, green. Both+, orange. Other, grey.

\section{Figure 3}

A
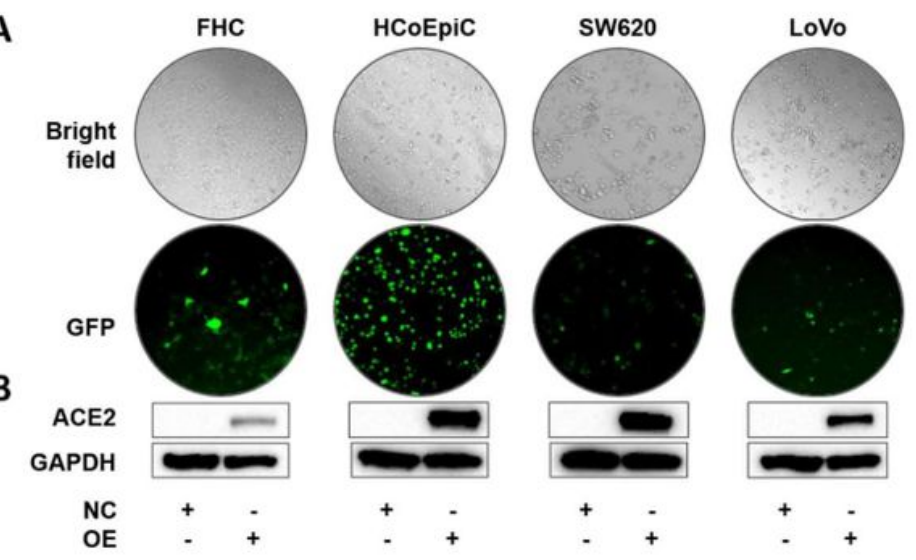

C

D
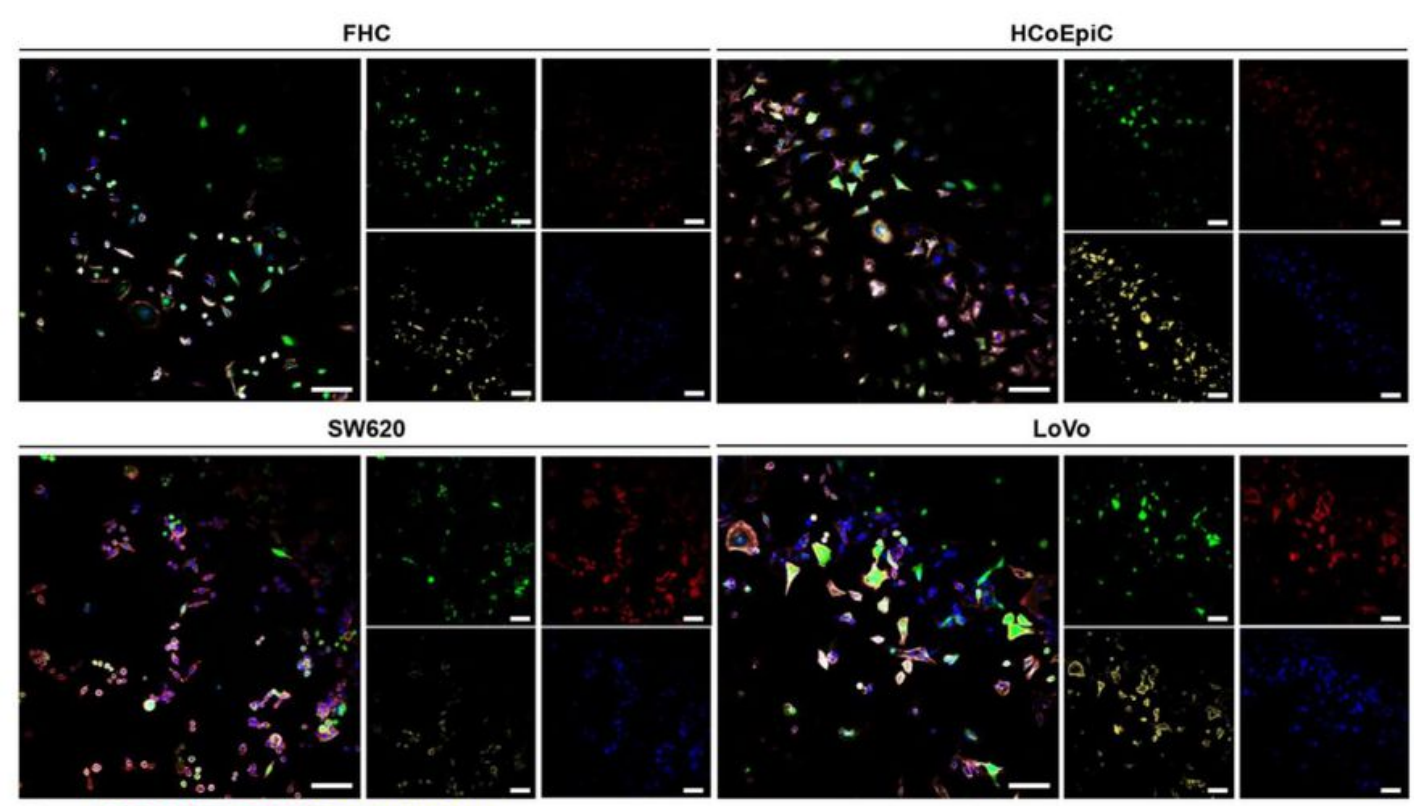

Merge/ACE2/SARS-CoV-2/F-actin/Nucleus

\section{Figure 3}

Infection with SARS-CoV-2 in ACE2-positive colon cell lines. (A) Transduction efficiency confirmed by the observation of GFP under a fluorescence microscope. Original magnification was 100x. (B) Stable overexpression of ACE2 in the respective cell lines was checked by Western blotting. (C) Luciferase reporter assay to measure SARS-CoV-2 infection in the respective cell lines. ${ }^{*} P<0.05$. (D) Representative 
images of the immunofluorescence staining of SARS-CoV-2-infected cells with stable overexpression of ACE2. Cells were fixed and stained for the ACE2 vector (GFP, green), SARS-CoV-2 S (mCherry, red), F-actin (phalloidin, yellow), and nucleus (DAPI, blue). Original magnification was 100x. Scale bars, $100 \mu \mathrm{m}$.

Figure 4
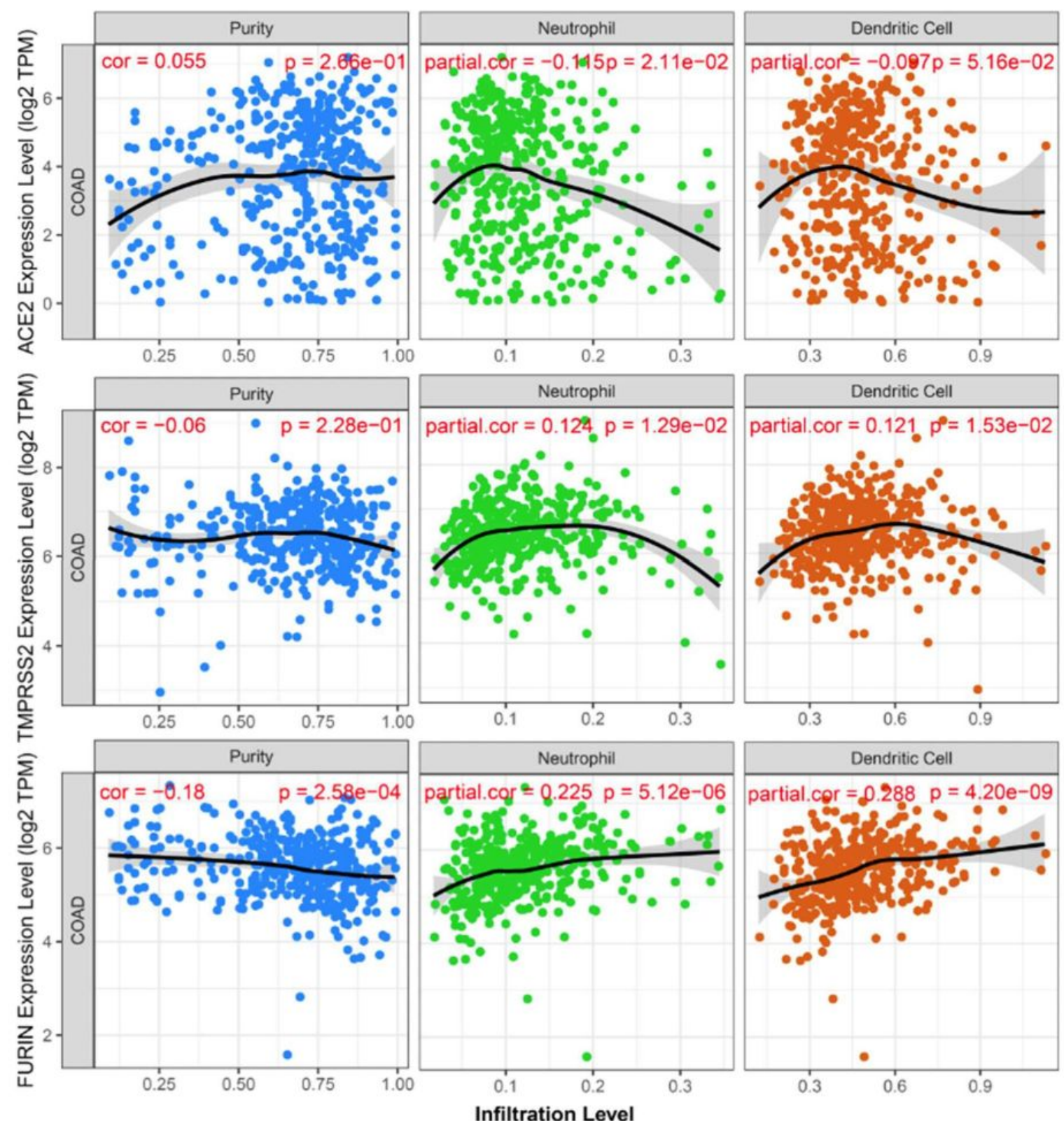

\section{Figure 4}

The correlation of ACE2 and co-factor expression with immune infiltration in colorectal cancer tissues. The levels of immune infiltration in ACE2 (top), TMPRSS2 (centre) and FURIN (bottom) obtained from the TIMER database. 
Figure 5
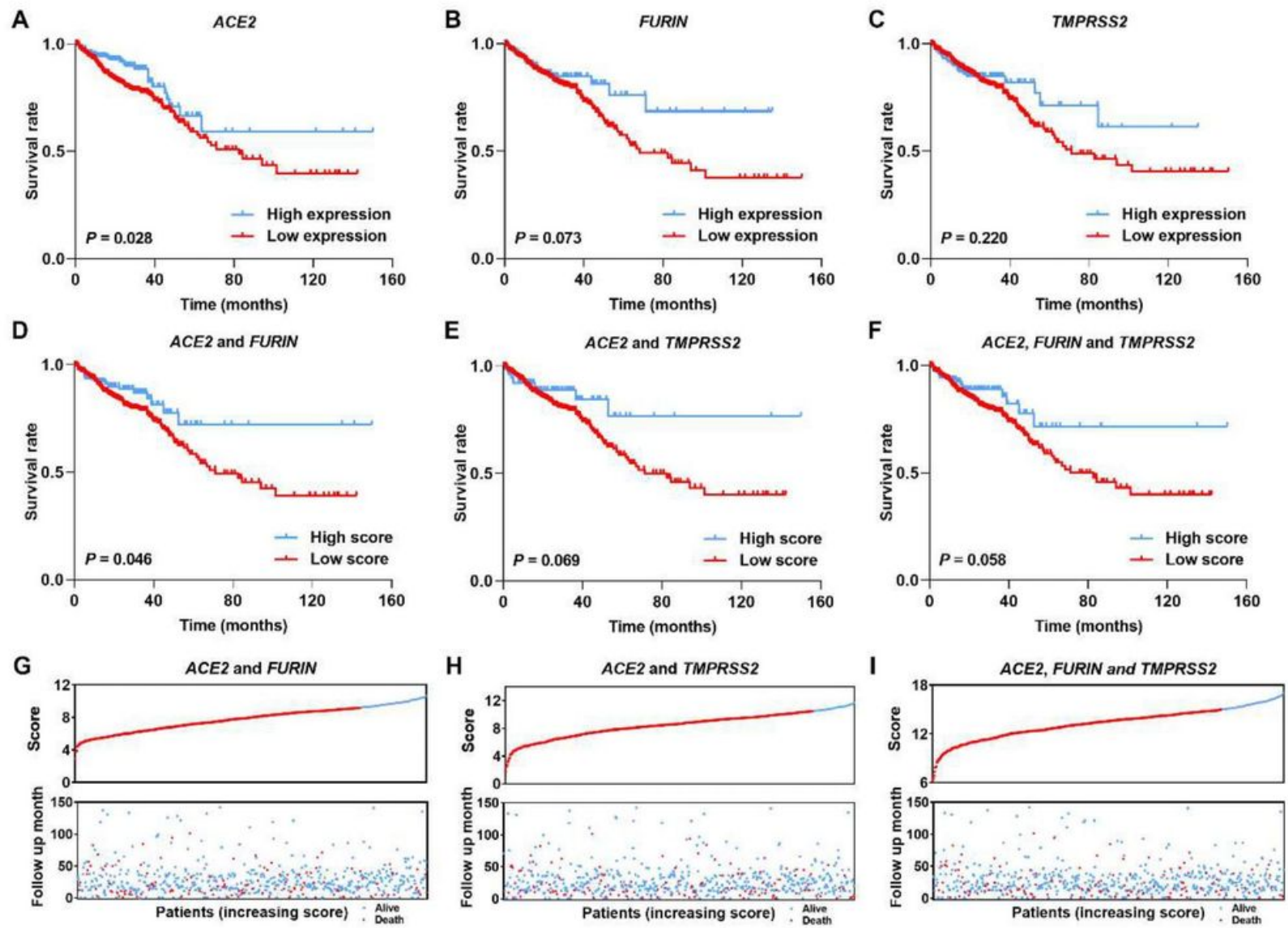

\section{Figure 5}

The performance of the prognostic model in predicting the overall survival of colorectal cancer patients. (A-C) Kaplan-Meier survival analysis of ACE2 (A), FURIN (B) and TMPRSS2 (C). (D-F) Patients were divided into low and high expression groups according to the median expression of selected genes. Kaplan-Meier survival analysis of ACE2 and FURIN (D), ACE2 and TMPRSS2 (E) and all three genes (F). Patients were divided into high and low score groups based on the median risk score under different combinations of selected genes. (G-I) The relationship between survival status, risk score rank (upper) and survival time (bottom). 


\section{Figure 6}

A

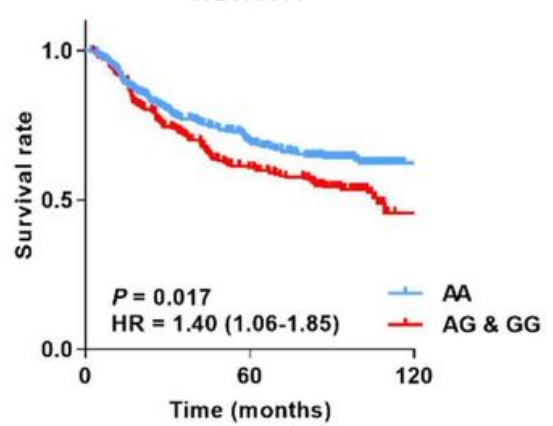

B

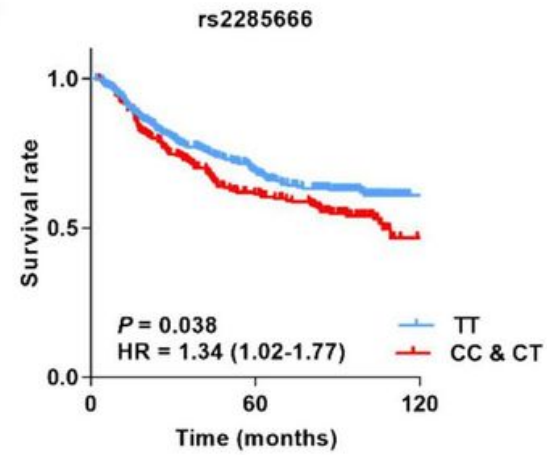

C

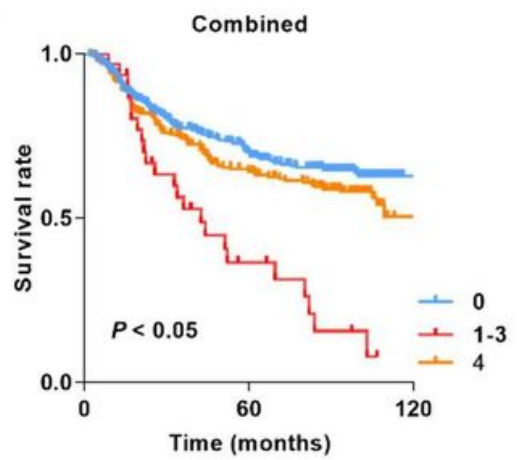

D

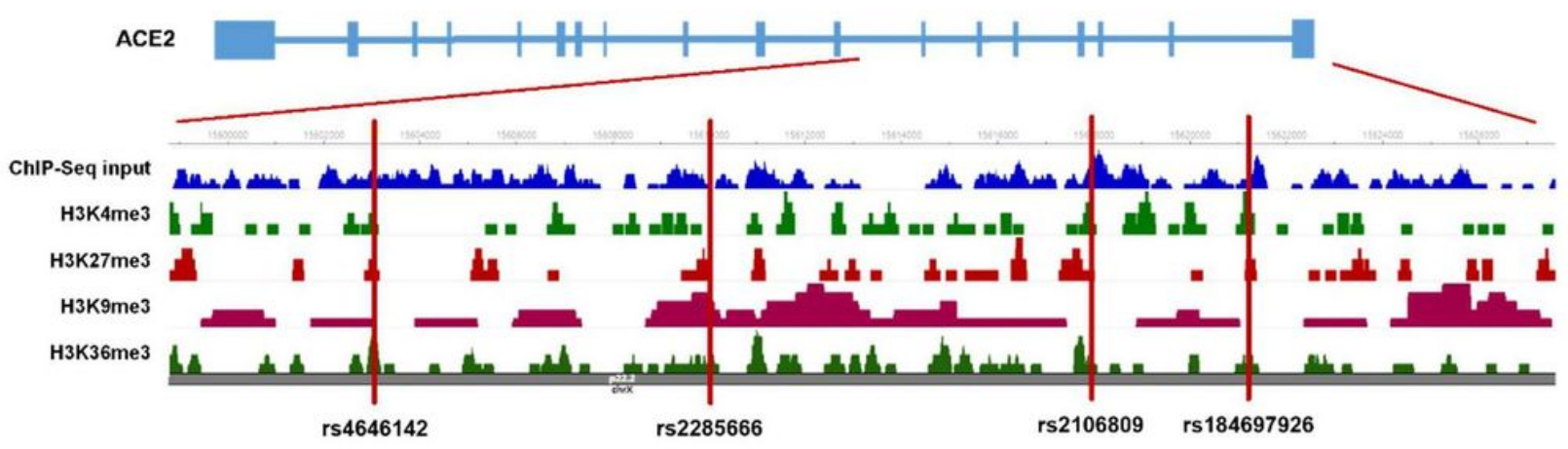

Figure 6

Comparison of Kaplan-Meier survival curves of genetic variants in the ACE2 gene in colorectal cancer. (AC) Kaplan-Meier survival analysis of rs2106809 (A) and rs2285666 (B) and the combined risk allele of two SNPs (C). (D) Enrichment of the ChIP-Seq input and histone marks in the regions that included candidate SNPs.

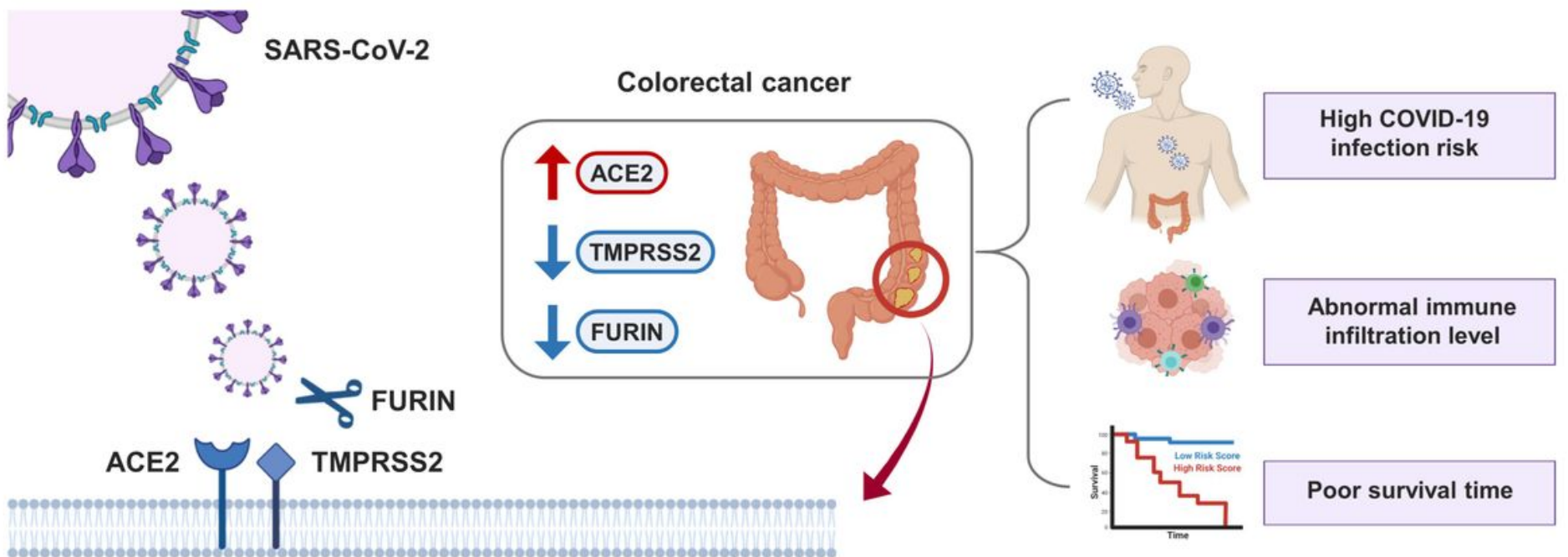

Tumor cell membrane 


\section{Figure 7}

Schematic representation of SARS-CoV-2 infection in colorectal cancer patients. ACE2 is upregulated in colorectal tumor tissues with the downregulation of TMPRSS2 and FURIN. After the stable overexpression of ACE2, the infection rate of SARS-CoV-2 in colorectal tumor cells was significantly higher than that in normal colon epithelial cells. These three genes play specific roles in abnormal immune infiltration in colorectal cancers and the poor survival time of patients. The diagram was constructed with BioRender (https://biorender.com/).

\section{Supplementary Files}

This is a list of supplementary files associated with this preprint. Click to download.

- Supplementaryfiles.docx 